\title{
Importance of Extubation Stress following Off-pump CABG
}

\author{
Fatih AYGÜN \\ Başkent University, Konya Medical and Research Center, Cardiovascular Surgery, Konya, TÜRKIYYE
}

\begin{abstract}
Corresponding Author:
Dr. Fatih AYGÜN

Email: drfatihaygun@gmail.com

This is an Open Access article distributed under the terms of the Creative Commons Attribution License (creativecommons.org/ licenses/by/3.0).
\end{abstract}

Received : October 12, 2017

Accepted : January 30, 2018

Published : February 15, 2018

\begin{abstract}
Background: Coronary artery bypass grafting $(\mathrm{CABG})$ is a frequently performed surgery worldwide. The classical coronary bypass surgery performed with the heart stopped and using heart-lung machine is called as on-pump CABG, whereas the coronary bypass surgery performed without stopping the heart or using heart-lung machine is called as off-pump CABG. It is thought that generally respiratory complications can occur during extubation stress in the patients undergoing off-pump CABG performed with fast-track protocol. Case Report: We report a 56 year old patient with $75 \%$ stenosis in left anterior descending and circumflex artery. He developed bradycardia after coronary artery bypass grafting by offpump technique. His repeat angiography showed stenotic right coronary artery after than it was corrected by percutaneous transluminal coronary angioplasty and stent implantation Conclusion: The probability of development of different clinical situations should be kept in mind and the patient should be re-explored without hesitating in case of any hemodynamic instability. Herein, I intended to discuss a different specific case.
\end{abstract}

Keywords: Angiography, Bradycardia, Coronary Artery Bypass, Heart, Stents.

\section{Introduction}

Coronary artery bypass grafting $(\mathrm{CABG})$ is a frequently performed surgery worldwide. It is performed in the patients who have coronary arteries with serious stenosis ( $75 \%$ and over) in appropriate localization detected after coronary angiography.

The classical coronary bypass surgery performed with the heart stopped and using heart-lung machine is called as on-pump CABG, whereas the coronary bypass surgery performed without stopping the heart or using heart-lung machine is called as off-pump CABG [1]. Traditional approach for the anesthesia for cardiac surgery includes high-dose opioid use and long-acting muscle relaxants. Fasttrack method was developed to reduce both duration of staying in intensive care unit (ICU) and the cost [24]. By this method, patients can be extubated within six hours following cardiac surgery.
It is thought that generally respiratory complications can occur during extubation stress in the patients undergoing off-pump CABG performed with fast-track protocol. The probability of development of different clinical situations should be kept in mind and the patient should be re-explored without hesitating in case of any hemodynamic instability. Herein, I intended to discuss a different specific case.

\section{Case Report}

A 56-year-old male patient visited the cardiology polyclinic with chest pain. His anamnesis revealed compression-like chest pain for one month with increasing intensity on exertion, which occurs also at resting. It was learned that he has not been receiving any medication, had no family history of coronary artery disease and no hyper-lipidemia, diabetes or hypertension. He has been smoking two packages of cigarettes per day and consuming approximately $20 \mathrm{~mL}$ alcohol per day. 
On his physical examination, body temperature was $36.7^{\circ} \mathrm{C}$, arterial blood pressure was $130 / 70 \mathrm{mmHg}$, respiratory rate was $16 / \mathrm{min}$, pulse rate was $83 / \mathrm{min}$, and oxygen saturation by pulse-oximeter was $90-93 \%$. Heart sound was rhythmic with normal S1-S2 without abnormal sound or murmur. It was determined that each hemi-thorax moves symmetrically, and no rale or rhoncus was heard. Abdominal examination revealed no distension, rebound or tenderness. Peripheral pulses were palpable. With regard to the cardiac markers in the laboratory analyses, troponin-I was normal and CK-MB was mildly elevated (28 mmol/L; reference range: 0-25 $\mathrm{mmol} / \mathrm{L})$. On his electrocardiography, negative $\mathrm{T}$ waves were detected in the anterior leads. While his trans-thoracic echocardiography performed in the polyclinic demonstrated no valvular pathology, decreased ventricular systolic functions were determined. The patient was admitted to the coronary angiography (CAG) laboratory for percutaneous coronary intervention. After CAG, stenosis greater than $75 \%$ was detected in the branching point of proximal and diagonal left anterior descending (LAD) and in the circumflex $(\mathrm{Cx})$ coronary artery [Fig.1], whereas right coronary artery had stenosis not requiring surgery [Fig.2,3].

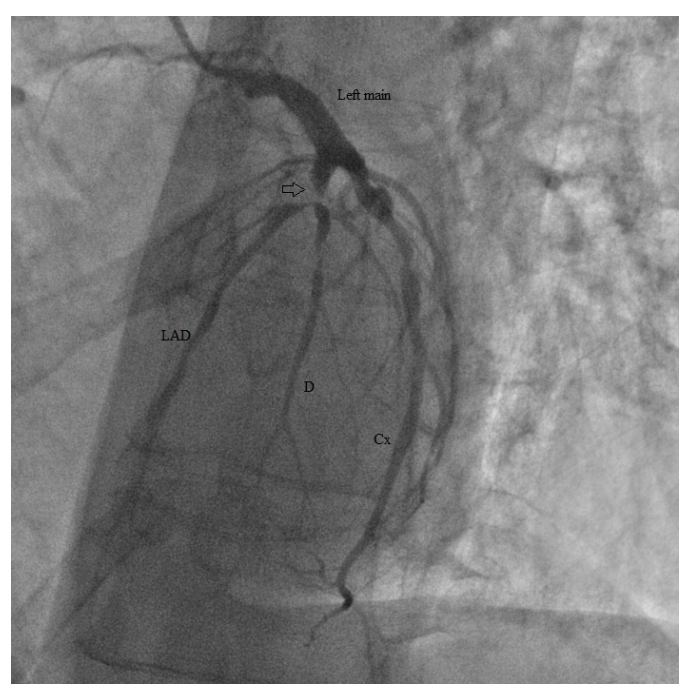

Fig.1: Pre-operative left anterior descending-circumflex artery.
CABG was declared to the patient and the consent of his relatives was obtained. He was pre-medicated and admitted to the cardiovascular surgery clinic for $\mathrm{CABG}$ with priority. Premedication started with metoprolol (50 mg oral pill twice daily), enoxaparin sodium (8000 anti-Xa IU twice daily via subcutaneous route), acetyl salicylic acid (100 mg oral pill, daily), and nitroglycerin infusion ( $2 \mathrm{ml} /$ hour in solution).

The patient was admitted to the operation room. CABG (LIMA-LAD, Ao-saphenous-D; Ao-saphenous-Cx) was performed by off-pump (beating heart) technique. At the end of surgery,

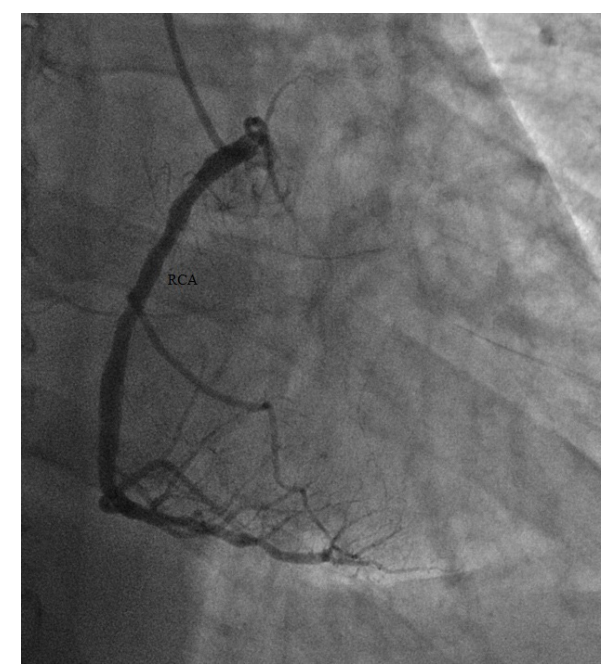

Fig.2: Pre-operative right coronary artery.

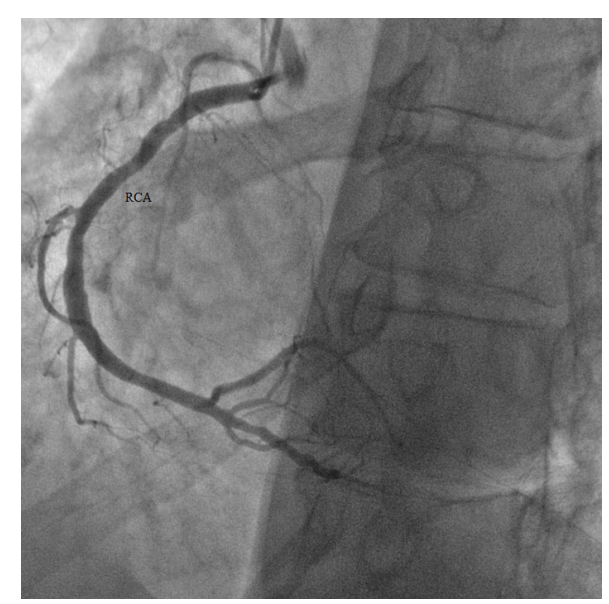

Fig.3: Pre-operative right coronary artery. 
activated clotting time (ACT) was given within 150-200 seconds and nitroglycerine infusion was administered before admitting the patient to the cardiovascular ICU. The patient woke up earlier because fast-track anesthesia has been performed. Preparations for early extubation were started as the post-operative mediastinal drainage was normal and hemodynamics and respiratory functions were suitable for extubation. The patient developed sudden bradycardia (nodal rhythm) as soon as he was extubated and his blood pressure decreased to $70 / 40 \mathrm{mmHg}$. Because of hemodynamic instability, the patient was rapidly admitted to the operation room and re-explored. It was determined that there is no hematoma in the mediastinum, bypass in the left coronary artery system is working and left ventricular contraction is bradycardic but good. The only pathology observed was the right ventricular dilatation and bradycardia. Intraoperative trans-esophageal echocardiography showed no pathology except for the echological signs of the pathology determined by inspection. Although the heart and hemodynamic recovered somewhat by positive inotrope and pacemaker applications, it was decided to admit the patient to the coronary angiography laboratory and to visualize the right coronary artery. Since the heart outgrew the mediastinum, sternum could not be approximated and covered with sterile materials. The patient underwent coronary angiography in the angiography laboratory. It was determined that the right coronary artery is completely stenotic [Fig.4]. After detecting the pathology, percutaneous transluminal coronary angioplasty (PTCA) and stent implantation was performed in the RCA [Fig.5-6]. The patient was admitted to the cardiovascular ICU after the procedure. Hemodynamics recovered within the first 24 hours. Sternum was approximated in the operation room because the heart returned to the sinus rhythm, dopamine infusion was under 3 microgram $/ \mathrm{kg} /$ $\mathrm{mL}$, and hemodynamic was stable. The patient was extubated in the next 24 hours. As the clinical picture of the patient improved, he was discharged

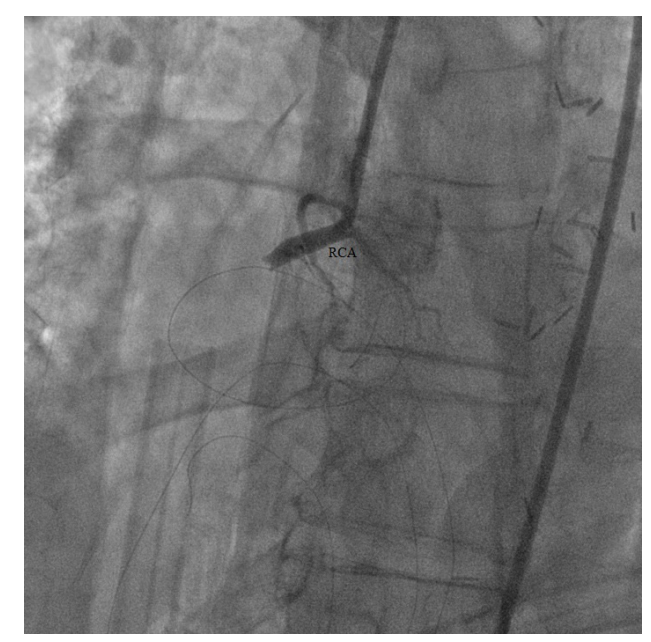

Fig.4: Post-operative right coronary artery.

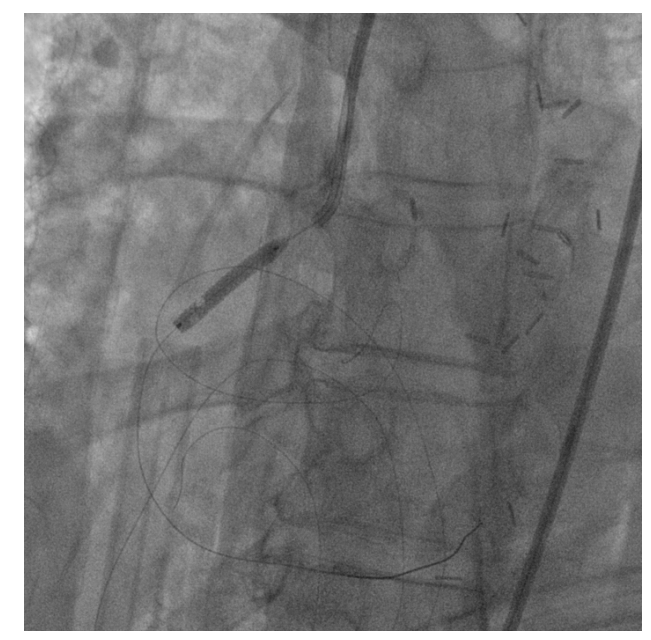

Fig.5: Post-operative right coronary artery.

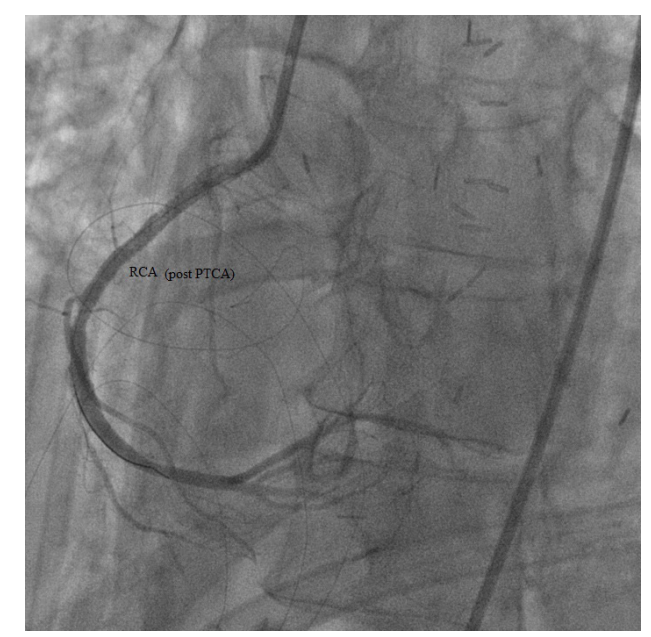

Fig.6: Right coronary artery, post-percutaneous transluminal coronary angioplasty (PTCA). 
on the postoperative $7^{\text {th }}$ day after routine $C A B G$ monitoring and applications.

\section{Discussion}

Recently, many patients with gradually increasing high-risk profile have had to undergo CABG. The benefits of off-pump CABG in terms of CPBand aorta-related complications (inflammation, negative effect on blood and coagulation factors) are clear. Recent studies have demonstrated the progresses in high-risk patients that had undergone off-pump CABG [5-8].

Early extubation is a method performed in suitable conditions to improve cardiac output, provide painless situation, remove the airway and lung trauma, and to make the patient more stable particularly in off-pump CABG patients $[9,10]$. Fast-track anesthesia protocol targets maximum cardiac protection and maintenance of stable rhythm over the course of off-pump CABG, perfect postoperative anesthesia, extubation within the postoperative 0-6 hours, and to shorten the duration of staying in ICU and hospital [11-13]. Fast-track anesthesia is safe and cost-effective [14].

Myocardial infarction (MI) results from the death (permanent injury) of a part of the cardiac muscle due to inadequate blood supply (usually results from complete obstruction of coronary artery) to the relevant region. Thrombi develop in the coronary arteries that have been constricted due to atherosclerotic changes. Atherosclerotic plaque in the arterial wall is sometimes cracked and this as well triggers thrombus formation. Most of the above-mentioned risk factors are associated with overweight. Thrombus formation in the coronary artery, which has been constricted due to atherosclerosis, may occur for any reason. Sometimes, sudden and high stress may lead to this.

\section{Conclusion}

Earlier studies have reported that blood and coagulation factors are not affected in the patients that underwent off-pump CABG. In case coronary artery disease is underestimated, a complication that might be developed despite high ACT level (150-200 seconds) in the coronary arteries, which contain plaque and have not undergone bypass procedure, could be overlooked. In the off-pump CABG cases, non-graftable coronary arteries with plaque are likely to be more susceptible to the extubation process as was the present case. In addition, re-exploration without hesitating in case of unusual events observed during patient monitoring after $\mathrm{CABG}$ is a critical approach in the name of prompt treatment.

Contributors: FA wrote the manuscript, did literature search, involved in patient management, and approved the final version of this article. He will act as guarantor of the study.

Funding: None; Competing interests: None stated.

\section{References}

1. Lassnigg A, Hiesmayr MJ, Bauer P, Haisjackl M. Effect of centre-,patient- and procedure-related factors on intensive care resource utilisation after cardiac surgery. Intensive Care Med. 2002;28:1453-1461.

2. Levy JH, Tanaka KA. Inflammatory response to cardiopulmonary bypass. Ann Thorac Surg. 2003;75:715720.

3. Møller CH, Penninga L, Wetterslev J, Steinbrüchel DA, Gluud C. Clinical outcomes in randomized trials of offvs. on-pump coronary artery bypass surgery: Systematic review with meta-analyses and trial sequential analyses. Eur Heart J. 2008;29:2601-2616.

4. Nuttall GA, Erchul DT, Haight TJ, Ringhofer SN, Miller TL, Oliver WC Jr, et al. A comparison of bleeding and transfusion in patients who undergo coronary artery bypass grafting via sternotomy with and without cardiopulmonary bypass. J Cardiothorac Vasc Anesth. 2003; 17:447-451.

5. Lemma MG, Coscioni E, Tritto FP, Centofanti P, Fondacone C, Salica A, et al. On-pump versus off-pump coronary artery bypass surgery in high-risk patients: operative results of a prospective randomized trial (onoff study). J Thorac Cardiovasc Surg. 2012;143:625631.

6. Barandon L, Richebé P, Munos E, Calderon J, Lafitte M, Lafitte $\mathrm{S}$, et al. Off-pump coronary artery bypass surgery in very high-risk patients: adjustment and preliminary results. Inte ract Cardiovasc Thorac Surg. 2008;7:789793. 
7. Marui A, Okabayashi H, Komiya T, Tanaka S, Furukawa $\mathrm{Y}$, Kita $\mathrm{T}$, et al. Benefits of off-pump coronary artery bypass grafting in high-risk patients. Circulation. 2012;126:151-157.

8. Mack MJ, Pfister A, Bachand D, Emery R, Magee MJ, Connolly $\mathrm{M}$, et al. Comparison of coronary bypass surgery with and without cardiopulmonary bypass in patients with multivessel disease. J Thorac Cardiovasc Surg. 2004;127:167-173.

9. Cheng DC. Routine immediate extubation in the operating room after OPCAB surgery: Benefits for patients, practitioners, or providers? J Cardiothorac Vasc Anesth. 2005;19:279-281.

10. Dorsa AG, Rossi AI, Thierer J, Lupiañez B, Vrancic $\mathrm{JM}$, Vaccarino GN, et al. Immediate extubation after off-pump coronary artery bypass graft surgery in 1,196 consecutive patients: Feasibility, safety and predictors of when not to attempt it. J Cardiothorac Vasc Anesth. 2011;25:431-436.

11. Hett DA. Anaesthesia for off-pump coronary artery surgery. Contin Educ Anaesth Crit Care Pain. 2006;6:6062.

12. Westaby S, Pillai R, Parry A, O'Regan D, Giannopoulos $\mathrm{N}$, Grebenik K, et al. Does modern cardiac surgery require conventional intensive care? Eur J Cardiothorac Surg. 1993;7:313-318.

13. White PF, Kehlet H, Neal JM, Schricker T, Carr DB, Carli F. The role of the anesthesiologist in fast-track surgery: From multimodal analgesia to perioperative medical care. Anesth Analg. 2007;104:1380-1396.

14. Myles PS, Daly DJ, Djaiani G, Lee A, Cheng DCH. A systematic review of the safety and effectiveness of fasttrack cardiac anesthesia. Anesthesiology. 2003;99:982987. 